\title{
Pinning Down the Fate of Fluorine
}

\author{
The first results from the Jinping Underground Nuclear Astrophysics \\ particle accelerator refine a key reaction rate for the destruction of \\ fluorine in stars.
}

By Christopher Crockett

$\prod$ he origin of fluorine is puzzling. The element is absent in the main nuclear reactions in stars, making it hard to figure out how it is formed. Fluorine is also easily destroyed by run-ins with protons and helium nuclei, destructive reactions whose contributions to fluorine's lifecycle have yet to be pinned down because of difficulties in measuring the requisite reaction rates. A new particle accelerator in China could help in solving that problem, as its first results provide sharply reduced uncertainties in one fluorine reaction, fluorine atoms and protons convert to oxygen and helium atoms and gamma rays [1]. While many of the details of fluorine's origin and fate remain a mystery, these new reaction rates will help refine ongoing calculations of this element's abundance in the cosmos.

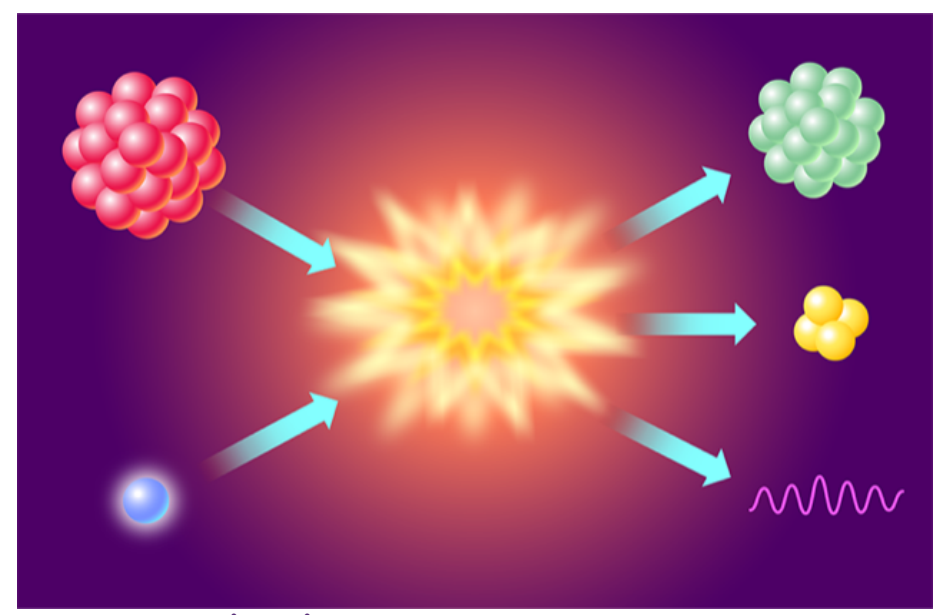

Credit: APS/Carin Cain
The Jinping Underground Nuclear Astrophysics (JUNA) experimental facility is a recent addition to the deepest operational particle physics lab in the world. Sitting beneath 2400 meters of rock, JUNA's accelerator is well shielded from the cosmic rays that have hindered other attempts to directly measure a particular transformation of fluorine to oxygen at the proton energies relevant to the interiors of stars.

For their inaugural experiment, researchers bombarded two fluorine targets with proton beams that had energies as a low as $76.2 \mathrm{keV}$-an unprecedently small value-and recorded the ensuing shower of gamma rays. From those measurements, they calculated that fluorine converts to oxygen via this reaction channel at a rate ranging from $1.23 \times 10^{-64} \mathrm{~cm}^{3} \mathrm{~s}^{-1} \mathrm{~mol}^{-1}$ to $1.29 \times 10^{+5} \mathrm{~cm}^{3} \mathrm{~s}^{-1} \mathrm{~mol}^{-1}$ depending on the reaction temperature. Over the temperature range of interest to astrophysics, the error in the measurements was below $10 \%$, down from orders of magnitude, because of the ultralow cosmic-ray background and high intensity of the proton beam.

Christopher Crockett is a freelance writer based in Arlington, Virginia.

\section{REFERENCES}

1. L. Y. Zhang et al., "Direct measurement of the astrophysical ${ }^{19} \mathrm{~F}(p, \alpha \gamma){ }^{16} \mathrm{O}$ reaction in the deepest operational underground laboratory," Phys. Rev. Lett. 127, 152702 (2021). 